Revista de Matemática: Teoría y Aplicaciones 4(1): 43-50 (1997)

\title{
TRIÁNGULOS CASI CONGRUENTES
}

\author{
Eduardo Piza Volio ${ }^{1}$
}

\begin{abstract}
Resumen
En este artículo se estudian los triángulos casi congruentes, que consisten en las parejas de triángulos que tienen exactamente 5 de sus 6 elementos congruentes. Se caracterizan todas las soluciones enteras a este problema. Se analizan algunas propiedades interesantes, concernientes al tipo de ángulos, a los perímetros, a las áreas y algunas otras. Se establecen algunas relaciones interesantes entre los triángulos casi congruentes y la famosa razón áurea.
\end{abstract}

\section{Introducción}

Cada triángulo tiene 6 elementos principales: sus tres lados y sus tres ángulos. ¿Será posible hallar una pareja de triángulos, de forma tal que en ellos coincidan 5 de sus 6 elementos principales, sin ser, pese a ello, congruentes ambos triángulos? He formulado esta pregunta a gran cantidad de amigos científicos — algunos de ellos matemáticos profesionales - y la respuesta invariablemente ha sido un ... ¡no!

Puede parecer imposible a primera vista, aunque resulta que sí existen tales parejas de triángulos casi congruentes, como los llamaremos. Más aún, existen infinidad de soluciones a este extraño y misterioso problema involucrando solamente lados enteros, como veremos a continuación.

\section{Existencia de soluciones}

Ya en la época de Euclides se estudiaron tres postulados de congruencia de triángulos en los que la congruencia de los mismos queda asegurada por la igualdad de ciertas ternas de elementos: (i) igualdad de dos lados y el ángulo comprendido entre ambos; (ii) igualdad de los tres lados; (iii) igualdad de un lado y los dos ángulos contiguos. Debe aclararse que en ciertos casos la congruencia de los dos triángulos se obtiene luego de una reflexión en el espejo.

De lo anterior se deduce que para obtener una pareja de triángulos casi congruentes es necesario desechar el caso en que hay igualdad entre los tres lados correspondientes de cada

\footnotetext{
${ }^{1}$ Centro de Investigaciones en Matemáticas Puras y Aplicadas (Cimpa), Escuela de MatemáticA, Universidad De Costa RicA, 2060 SAn José, Costa Rica. E-mail: epiza@cariari.ucr.ac.cr
} 
triángulo, pues el postulado (ii) nos llevaría a la congruencia total de ambos triángulos. Luego, la única posibilidad estriba en que los triángulos tengan iguales los tres ángulos y dos de sus lados, pero no precisamente los lados homólogos, pues los postulados (i) e (iii) nos llevarían de nuevo a la congruencia absoluta de ambos triángulos.

La situación anterior se ilustra en la Figura 1, en la cual los triángulos I y II tienen 5 de sus elementos principales iguales: los tres ángulos $(\alpha, \beta, \gamma)$ y dos de sus lados $(b, c)$. Sin embargo, difieren en el sexto elemento: $a$ en I y $d$ en II. Encontraremos condiciones necesarias y suficientes para poder construir de manera coherente tal pareja de triángulos.
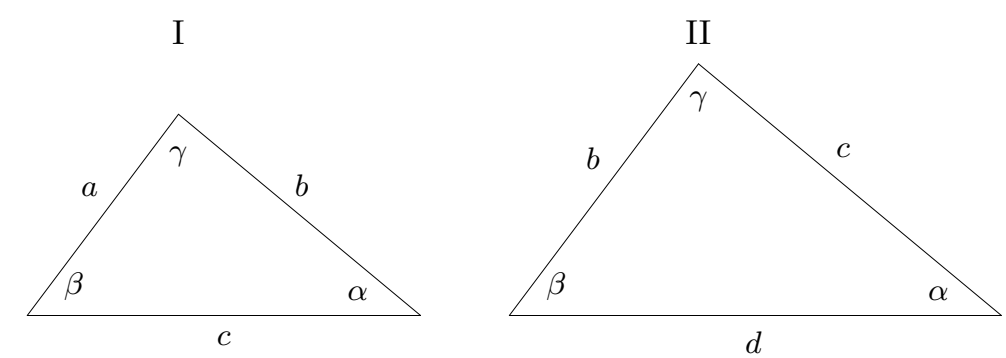

Figura 1: Condición necesaria para la existencia de los triángulos casi congruentes: hay 5 elementos principales iguales en I y II, y el sexto difiere.

En la Figura 1 hemos dispuesto los triángulos I y II de forma tal que los lados cumplan con las desigualdades $a<b<c<d$. En virtud de que ambos triángulos I y II son semejantes, entonces todos los cocientes entre dos de los lados correspondientes deben coincidir. Así obtenemos las siguientes condiciones necesarias:

$$
\frac{b}{a}=\frac{c}{b} \quad ; \quad \frac{c}{b}=\frac{d}{c}
$$

Si llamamos por $r$ el cociente $\frac{b}{a}$, entonces de (1) obtenemos las expresiones para los lados $b, c$ y $d$ en términos de $r$ y $a$ :

$$
b=a r \quad ; \quad c=a r^{2} \quad ; \quad d=a r^{3} .
$$

En (2) el valor de a puede ser cualquier número real positivo, mientras que hay ciertas restricciones para el generador $r$. En efecto, $r$ debe tratarse de un número real mayor que 1 , pues hemos supuesto que $a<b$. Surge además otra restricción propia de la naturaleza de los triángulos, cual es que la suma de dos cualquiera de los lados debe ser mayor que el lado restante. Debido a que $a<b<c$, la anterior restricción se puede resumir en la desigualdad $a+b>c$, la cual, expresada en términos de $r$ luce como $a+a r>a r^{2}$. Simplificando el factor a llegamos a la desigualdad de segundo grado $r^{2}-r-1<0$, cuya solución es $1<r<\phi$, donde $\phi=\frac{1+\sqrt{5}}{2}$ es la famosa razón áurea o proporción divina.

En resumen, las condiciones necesarias para la existencia de los triángulos casi congruentes son las siguientes: los tres ángulos son iguales y los lados cumplen con las restricciones $a<b<c<d$, donde $a$ es cualquier número real positivo, $b=a r, c=a r^{2}, d=a r^{3}$, siendo el número real $r$ el generador del cuarteto $(a, b, c, d)$, con la restricción $1<r<\phi$. Esto se ilustra en la Figura 2. 

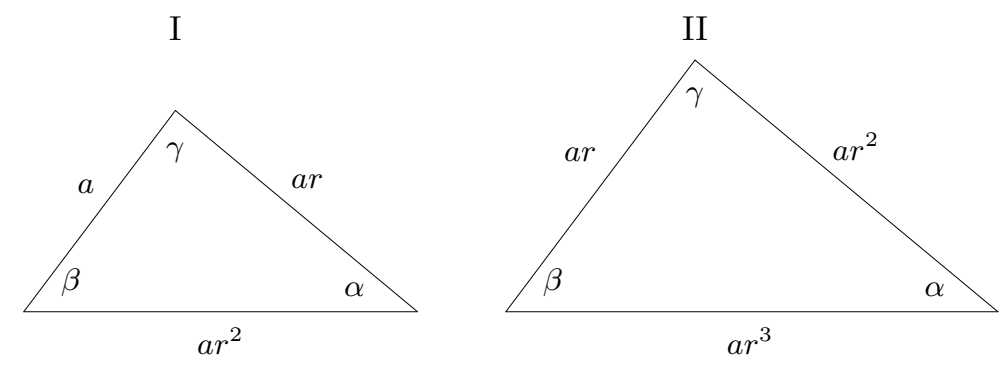

Figura 2: El lado a mide cualquier número real positivo. El generador $r$ debe satisfacer la desigualdad $1<r<\phi$, donde $\phi=(1+\sqrt{5}) / 2$ es la razón áurea.

El lector puede comprobar mediante la ley de los cosenos que efectivamente en los triángulos I y II de la Figura 2 los ángulos son iguales, de manera las condiciones arriba descritas son también suficientes.

\section{Soluciones enteras}

Buscaremos ahora todas las soluciones enteras de los triángulos casi congruentes, esto es, cuartetas de números enteros positivos $(a, b, c, d)$, irreducibles, que representen los lados de los triángulos I y II de la Figura 2. En este tipo de problemas, al igual que en el problema de las ternas pitagóricas ${ }^{2}$, interesa hallar todas las soluciones enteras.

Empecemos escogiendo $a$ como un entero positivo cualquiera. Debido a que $b=a r$ debe ser un numero entero, entonces necesariamente $r$ deberá ser un número racional, $r=n / m$ en forma irreducible. Además, $d=a r^{3}=a n^{3} / m^{3}$, y como $d$ debe ser entero se deduce que $m^{3}$ debe dividir a $a$ (nótese que $m^{3}$ y $n^{3}$ son primos relativos, por hipótesis). Escribiendo $a=\mathrm{km}^{3}$ entonces obtenemos, de (2):

$$
a=k m^{3} \quad ; \quad b=k m^{2} n \quad ; \quad c=k m n^{2} ; \quad d=k n^{3} .
$$

En virtud del requisito de la irreducibilidad del cuarteto $(a, b, c, d)$ obtenemos que $k=1$. Finalmente, las restricciones sobre el generador $(1<r<\phi)$ nos llevan a la desigualdad $0<m<n<m \phi$. Hemos demostrado el siguiente teorema:

Teorema 1 Los cuartetos $(a, b, c, d)$, correspondientes a los lados enteros de los triángulos casi congruentes I y II de la Figura 2, son exactamente todos aquellos de la forma

$$
a=m^{3} \quad ; \quad b=m^{2} n \quad ; \quad c=m n^{2} \quad ; \quad d=n^{3},
$$

donde $m$ y $n$ son enteros primos relativos que cumplen $0<m<n<m \phi$, con $\phi$ la razón áurea.

\footnotetext{
${ }^{2}$ Las ternas pitagóricas primitivas son aquellas ternas $(a, b, c)$ de números enteros positivos que satisfacen la relación pitagórica $a^{2}+b^{2}=c^{2}$, o sea, que representan los lados enteros de un triángulo rectángulo. Además se requiere que los lados $a, b, \mathrm{y} c$ no contengan factores comunes. Las ternas pitagóricas provienen todas la siguiente fórmula $[\operatorname{Rad} 90]: a=u^{2}-v^{2}, b=2 u v, c=u^{2}+v^{2}$, donde $u>v>0$ son dos números enteros primos relativos y de diferente paridad.
} 
A partir del teorema anterior podemos encontrar los primeros cuartetos, como se ilustra en la tabla de la Figura 3.

\begin{tabular}{|c|c|c|}
\hline Generador & Condiciones & Cuarteto \\
\hline $2 / 1=2$ & $1<2 \nless 1 \phi \approx 1.62$ & no califica \\
\hline $3 / 2=1.5$ & $2<3<2 \phi \approx 3.24$ & $8,12,18,27$ \\
\hline $4 / 3 \approx 1.33$ & $3<4<3 \phi \approx 4.85$ & $27,36,48,64$ \\
\hline $5 / 3 \approx 1.66$ & $3<5 \nless 3 \phi \approx 4.85$ & no califica \\
\hline $5 / 4=1.25$ & $4<5<4 \phi \approx 6.47$ & $64,80,100,125$ \\
\hline $5 / 2 \approx 2.33$ & $2<5 \nless 2 \phi \approx 3.24$ & no califica \\
\hline $6 / 5=1.2$ & $5<6<5 \phi \approx 8.09$ & $125,150,180,216$ \\
\hline $7 / 2=3.5$ & $2<7 \nless 2 \phi \approx 3.24$ & no califica \\
\hline $7 / 3 \approx 2.33$ & $3<7 \nless 3 \phi \approx 4.85$ & no califica \\
\hline $7 / 4=1.75$ & $4<7 \nless 4 \phi \approx 6.47$ & no califica \\
\hline $7 / 5=1.4$ & $5<7<5 \phi \approx 8.09$ & $125,175,245,343$ \\
\hline $7 / 6 \approx 1.16$ & $6<7<6 \phi \approx 9.71$ & $216,252,294,343$ \\
\hline
\end{tabular}

Figura 3: Tabla de los primeras parejas de triágulos casi congruentes.

\section{4 ¿Obtusángulos o acutángulos?}

Investiguemos ahora cuáles de los triángulos casi congruentes son acutángulos y cuáles son obtusángulos. Volviendo a la Figura 2, el ángulo mayor de cada triángulo es $\gamma$. Aplicando la ley de los cosenos, $c^{2}=a^{2}+b^{2}-2 a b \cos \gamma$, y luego

$$
\cos \gamma=\frac{a^{2}+b^{2}-c^{2}}{2 a b}=\frac{1+r^{2}-r^{4}}{2 r}
$$

donde $r$ es el generador de la pareja de triángulos. Ahora, los triángulos son acutángulos si y solo si el ángulo mayor $\gamma$ es menor que $\frac{\pi}{2}$, lo cual ocurre si y solo si $\cos \gamma>\cos \frac{\pi}{2}=0$, pues la función coseno es decreciente en $\left[0, \frac{\pi}{2}\right]$. Llegamos a una desigualdad bicuadrática de fácil solución analítica (recuérdese que $1<r<\phi$ ):

$$
\begin{aligned}
\cos \gamma>0 & \Longleftrightarrow 1+r^{2}-r^{4}>0 \\
& \Longleftrightarrow 1<r<\sqrt{\phi} \approx 1.2720
\end{aligned}
$$

lo cual demuestra el siguiente teorema.

Teorema 2 Los triángulos casi congruentes con generador $r$ son acutángulos si y solo si $1<r<\sqrt{\phi}$ y son obtusángulos si y solo si $\sqrt{\phi}<r<\phi$, donde $\phi$ es la razón áurea. 

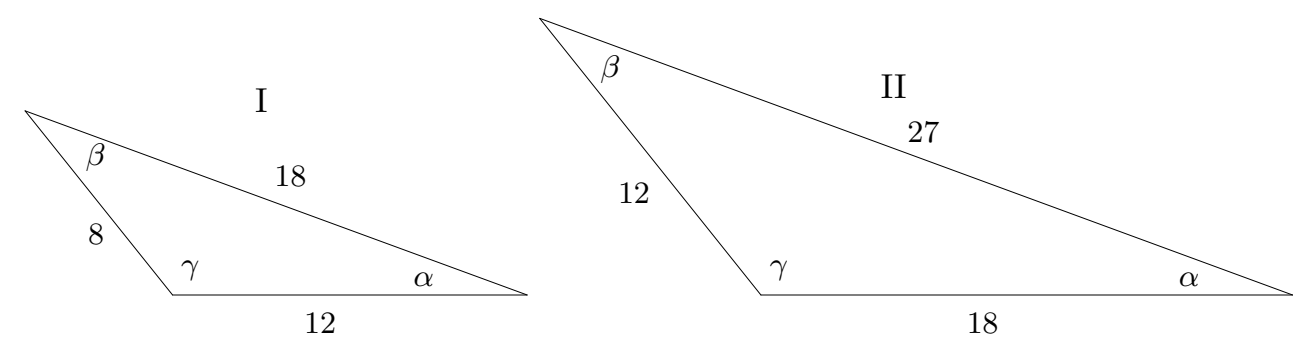

Figura 4: Primer cuarteto obtusángulo: $r=\frac{3}{2}>\sqrt{\phi}$.

Por ejemplo, la primera pareja de triángulos casi congruentes de la tabla de la Figura 3 corresponde a triángulos obtusángulos, pues $r=\frac{3}{2}=1.5>\sqrt{\phi}$. Estos triángulos se ilustran en la Figura 4.

La primera pareja de triángulos casi congruentes acutángulos corresponde a la quinta entrada de la tabla de la Figura 3, la cual se ilustra en la Figura 5.
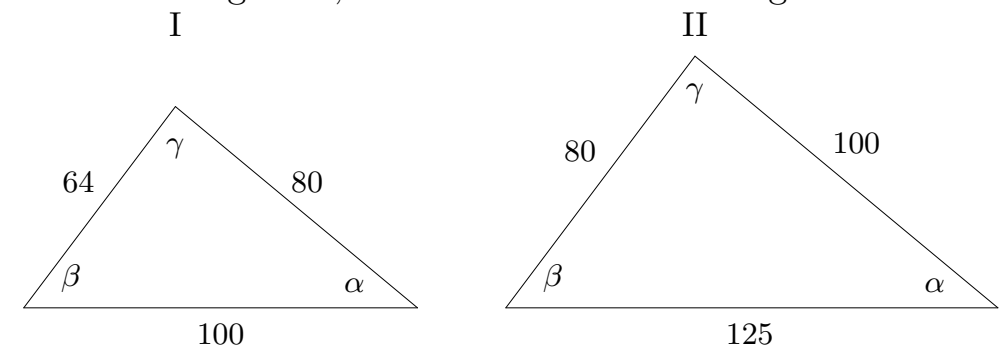

Figura 5: Primer cuarteto acutángulo: $r=\frac{5}{4}<\sqrt{\phi}$.

Una consecuencia de la demostración del Teorema 2 es el hecho de que no existen triángulos casi congruentes que sean rectángulos, con lados enteros. En efecto, los triángulos casi congruentes rectángulos corresponden al generador $r=\sqrt{\phi}$, que es un número irracional, el cual no genera soluciones enteras.

Interesante es considerar los triángulos producidos por generadores del tipo $r=\frac{n+1}{n}$, con $n \geq 4$. Es fácil comprobar que estos generadores siempre producen triángulos acutángulos, pues satisfacen las condiciones: $0<n<n+1<n \sqrt{\phi}$, para todo $n \geq 4$. Se trata de los generadores de los triángulos casi congruentes acutángulos máximos, en el sentido que el generador es muy cercano a 1 para valores grandes de $n$. Calculando para tal generador el coseno del ángulo máximo $\gamma$, obtenemos

$$
\begin{aligned}
\cos \gamma & =\frac{1+\left(\frac{n+1}{n}\right)^{2}-\left(\frac{n+1}{n}\right)^{4}}{2\left(\frac{n+1}{n}\right)} \\
& =\frac{n^{4}-2 n^{3}-5 n^{2}-4 n-1}{2 n^{4}+2 n^{3}},
\end{aligned}
$$

expresión última que tiende a $\frac{1}{2}$ cuando $n$ tiende a $+\infty$, lo cual corresponde al triángulo equilátero: se le puede considerar el extremo de los triángulos casi congruentes acutángulos. 


\section{Perímetros y áreas}

Para una pareja de triángulos casi congruentes, ¿Qué relación hay entre los perímetros de ambos triángulos? ¿Qué relación tienen las áreas?

Refiriéndonos de nuevo a la Figura 2, vemos que el cociente de los perímetros de los triángulos II y I es fácil de calcular:

$$
\frac{P_{I I}}{P_{I}}=\frac{a r+a r^{2}+a r^{3}}{a+a r+a r^{2}}=r
$$

Para comparar las áreas utilizamos la fórmula de Herón:

$$
\frac{A_{I I}}{A_{I}}=\left\{\frac{S_{I I}}{S_{I}} \cdot \frac{\left(S_{I I}-b\right)}{\left(S_{I}-a\right)} \cdot \frac{\left(S_{I I}-c\right)}{\left(S_{I}-b\right)} \cdot \frac{\left(S_{I I}-d\right)}{\left(S_{I}-c\right)}\right\}^{1 / 2},
$$

donde $S_{I I}$ y $S_{I}$ son los semi-perímetros de los triángulos II y I respectivamente. De los datos de la Figura 2 obtenemos las siguientes expresiones para cada término de la fórmula (4):

$$
\begin{aligned}
S_{I} & =\frac{a}{2}\left(r^{2}+r+1\right) & S_{I I} & =\frac{a r}{2}\left(r^{2}+r+1\right) \\
\left(S_{I}-a\right) & =\frac{a}{2}\left(r^{2}+r-1\right) & \left(S_{I I}-b\right) & =\frac{a r}{2}\left(r^{2}+r-1\right) \\
\left(S_{I}-b\right) & =\frac{a}{2}\left(r^{2}-r+1\right) & \left(S_{I I}-c\right) & =\frac{a r}{2}\left(r^{2}-r+1\right) \\
\left(S_{I}-c\right) & =\frac{a}{2}\left(-r^{2}+r+1\right) & \left(S_{I I}-d\right) & =\frac{a r}{2}\left(-r^{2}+r+1\right)
\end{aligned}
$$

Luego, el cociente de áreas $A_{I I} / A_{I}$ simplifica a $r^{2}$. Lo anterior se resume en el siguiente teorema.

Teorema 3 Para los triángulos casi congruentes con generador $r$ como en la Figura 2, el cociente de los perímetros $P_{I I} / P_{I}$ es igual a $r$, mientras que el cociente de las áreas $A_{I I} / A_{I}$ es igual a $r^{2}$.

\section{Tetrahedro de caras casi congruentes}

Interesante es el siguiente problema, el cual tiene relación con los triángulos casi congruentes:

Encontrar un tetrahedro ${ }^{3}$ cuyas caras sean todas triángulos escalenos con lados enteros, semejantes dos a dos pero no todas congruentes, con la restricción de que el lado (arista) mayor no exceda a 50. Describir las caras del tetrahedro.

El problema fue publicado en el Journal of the Assistant Master Association, en febrero de 1966 (pág. 75). Es muy interesante saber que la solución fue hallada por ... i un estudiante de colegio de diecisiete años! [Hun70]. La solución del muchacho se presenta en el Figura 6.

\footnotetext{
${ }^{3}$ Los tetrahedros son las pirámides de base triangular.
} 


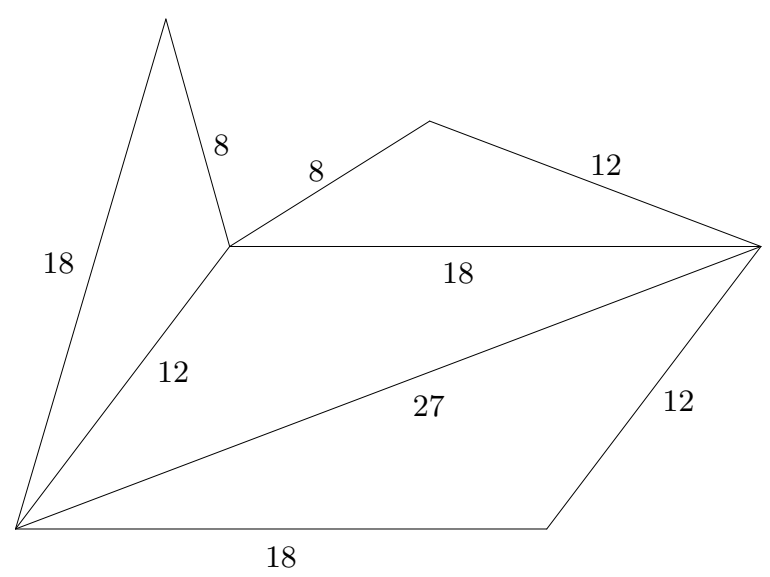

Figura 6: Tetrahedro entero de caras casi congruentes.

\section{Conclusiones}

Los resultados acerca de las soluciones enteras para los triángulos casi congruentes, así como las condiciones referentes al tipo de ángulos de los mismos (acutángulos u obtusángulos) y las comparaciones de perímetros y áreas fueron deducidas por el autor de manera independiente en 1994.

Sin embargo, las fórmulas acerca de las soluciones enteras ya habían sido estudiadas y deducidas por varios autores muchos años atrás en este siglo, algunos en forma independiente, entre ellos [Sal54], [The55], [Jon74]. Se ignora si el problema era conocido o si fue resuelto con anterioridad al año 1954, aunque es muy posible que se trate de esos famosos problemas que se olvidan en el tiempo y se redescubren muchos años más tarde.

Los triángulos casi congruentes constituyen un material didáctico de posible interés para los profesores de enseñanza media, pues su contenido es perfectamente accesible a los estudiantes de liceos y colegios. En particular, se trata una bella aplicación de los conceptos y principios de semejanza de triángulos.

\section{Referencias}

[Gra59] Graham, L. A. (1959) Ingenious Mathematical Problems and Methods. Dover, Problema 59.

[Hun70] Huntley, H. E. (1970) The Divine Proportion. Dover.

[Jon74] Jones, R. T.; Peterson, B. B. (1974) "Almost congruent triangles", en Mathematics Magazine, 47: 180-189.

[Rad90] Radamacher, H.; Toeplitz, O. (1990) The Enjoyment of Mathematics. Dover.

[Sal54] Salkind, C. (1954) "More than similar triangles", en Mathematical Teacher, 47: $561-562$. 
[The55] Thebault, V. (1955) "Problem E 1162", en American Mathematical Monthly, 62: $126,729-730$. 